\title{
Characterization of dysphagia following anterior cervical spine surgery
}

\author{
John P. Ziegler ${ }^{\mathrm{a}}$, Kate Davidson ${ }^{\mathrm{a}}$, Rebecca L. Cooper ${ }^{\mathrm{b}}$, Kendrea L. Garand ${ }^{\mathrm{c}}$, Shaun A. Nguyen ${ }^{\mathrm{a}}$, \\ Erick Yuen $^{\mathrm{a}}$, Bonnie Martin-Harris ${ }^{\mathrm{d}}$ and Ashli K. O’Rourke ${ }^{\mathrm{a}, *}$ \\ ${ }^{\mathrm{a}}$ Medical University of South Carolina, Charleston, SC, USA \\ ${ }^{\mathrm{b}}$ Orlando Healthcare Systems, Orlando, FL, USA \\ ${ }^{\mathrm{c}}$ University of South Alabama, Mobile, AL, USA \\ ${ }^{\mathrm{d}}$ Northwestern University, Evanston, IL, USA
}

Received 25 July 2021

Accepted 15 September 2021

\begin{abstract}
.
BACKGROUND: Post-operative dysphagia is one of the most common complications of anterior cervical spine surgery (ACSS).

OBJECTIVE: Examine post-operative structural and physiologic swallowing changes in patients with dysphagia following ACSS as compared with healthy age and gender matched controls.

METHODS: Videofluoroscopic swallow studies of adults with dysphagia after ACSS were retrospectively reviewed. Seventyfive patients were divided into early ( $\leq 2$ months) and late ( $>2$ months) post-surgical groups. Modified Barium Swallow Impairment Profile (MBSImP), Penetration-Aspiration Scale (PAS) scores, and pharyngeal wall thickness (PWT) metrics were compared.

RESULTS: Significant differences were identified for all parameters between the control and early post-operative group. MBSImP Pharyngeal Total (PT) scores were greater in the early group (Interquartile Range $(\mathrm{IQR})=9-14$, median $=12)$ versus controls $(4-7,5, P<0.001)$ and late group $(0.75-7.25,2, P<0.001)$. The early group had significantly higher maximum PAS scores (IQR $=3-8$, median $=7)$ than both the control group $(1-2,1, P<0.001)$ and late post-operative group (1-1.25, $1, P<0.001)$. PWT was significantly greater in the early $(\mathrm{IQR}=11.12-17.33 \mathrm{~mm}$, median $=14.32 \mathrm{~mm})$ and late groups (5.31-13.01, 9.15 mm) than controls $(3.81-5.41,4.68 \mathrm{~mm}, P<0.001)$.

CONCLUSION: Dysphagic complaints can persist more than two months following ACSS, but often do not correlate with validated physiologic swallowing dysfunction on VFSS. Future studies should focus on applications of newer technology to elucidate relevant deficits.
\end{abstract}

Keywords: Dysphagia, swallowing disorders, adults, fluoroscopy, spinal fusion, healthy

\section{Introduction}

Post-operative dysphagia is one of the most common complications of anterior cervical spine surgery (ACSS) with a majority of patients (71\%) reporting

\footnotetext{
*Corresponding author: Ashli O'Rourke, MD, Medical University of South Carolina, 135 Rutledge Ave MSC 550, Charleston, SC 29425, USA. Tel. :+1 843 7927162; E-mail: aorourke@musc.edu.
}

dysphagia symptoms within 2 weeks of surgery (Rihn et al., 2011). Likely contributing factors include prevertebral soft tissue swelling or altered sensation secondary to nerve traction during surgery (Anderson \& Arnold, 2013). Risk factors have been somewhat debated in the literature, with numerous studies investigating possible correlations between post-ACSS dysphagia and patient factors (i.e., age, gender, body mass index), surgical factors (i.e., operative time, use of instrumentation, highest level of surgery, number 
of levels, revision versus primary surgery), as well as the presence of pre-operative dysphagia (Anderson \& Arnold, 2013; Liu et al., 2017; Frempong-Boadu et al., 2002; Kang et al., 2016). Specific factors shown in the literature to be associated with increased risk of dysphagia include a greater number of vertebral levels addressed surgically, female sex, increased operative time and older age ( $>60$ years) (Anderson \& Arnold, 2013). Interestingly, intraoperative nerve monitoring has not been shown to reduce the complication rates following ACSS (Kilburg et al., 2006) and there is sparse literature on the effect of handedness of the surgeon and differences between right versus left surgical approach (Beutler et al., 2001; Badhiwala et al., 2019). Taken as a whole, however, these findings have proven to be largely inconsistent across studies as a recent meta-analysis precluded any firm conclusions regarding risk factors for post-operative ACSS dysphagia (Shriver et al., 2017). Therefore, although post-operative dysphagia following ACSS is well-represented in the literature, the underlying etiology of dysphagia following ACSS is poorly understood.

One potential reason contributing to the poor understanding of causal factors related to postoperative dysphagia risk is the lack of an operational definition and standardized method for determining the presence, severity, and nature of swallowing impairment, which precludes performing more robust analysis (Shriver et al., 2017; Riley et al., 2010). For example, current studies frequently utilize screening questionnaires and patient-reported outcome tools, most commonly the Bazaz dysphagia score (Bazaz et al., 2002) and the EAT-10 tool (Belafsky et al., 2008), to identify and evaluate the patient's perception of the severity of their dysphagia (Riley et al., 2010; Rosenthal et al., 2016). Despite their widespread use, these patient-reported questionnaires have more recently undergone scrutiny of their psychometric properties, and thus, their clinical utility remains questionable (Shriver et al., 2017; Wilmskoetter et al., 2019). Furthermore, asymptomatic dysphagia in this patient population has been reported and patient-reported outcome measures do not adequately capture the presence, nor the underlying nature of the swallowing impairment in order to effectively inform treatment decisions (Kang et al., 2016). This results in the majority of the post-ACSS dysphagia literature reporting little to no examination of the underlying pathophysiologic impairments contributing to overt swallowing dysfunction experienced by the patient (Muss et al., 2017).
The videofluoroscopic swallow study (VFSS), also referred to as the modified barium swallow study, is a comprehensive evaluation of oropharyngeal swallowing function (Martin-Harris et al., 2008; Martin-Harris et al., 2000). The VFSS allows for real-time radiographic imaging of bolus flow through the upper aerodigestive tract, which can provide insight into the underlying physiologic manifestations of swallowing impairment that can contribute to impaired airway safety and efficiency (Muss et al., 2017; Martin-Harris et al., 2008; MartinHarris et al., 2000). Despite these strengths, there are few studies that have employed VFSS to analyze swallowing physiology in patients with dysphagia following ACSS (Frempong-Boadu et al., 2002; Kang et al., 2016; Martin et al., 1997; Leonard \& Belafsky, 2011; Smith-Hammond et al., 2004). Although studies have contributed to our current knowledge regarding abnormal swallowing physiology occurring post-operatively and can guide clinical management, there are crucial limitations to consider. For example, the limited VFSS reports available varied in their approach, including weight/solution of barium administered, bolus consistency and volumes, and number of trials performed during the procedure. Further, interpretation of swallowing performance varied as to outcome measures reported, as well as the validity and reliability of such measures. The purpose of this investigation was to examine changes in structural, physiologic, and airway invasion swallowing measures in patients with dysphagia following ACSS. To account for variations in swallow function that may occur between sexes and/or resulting from typical aging alterations, each patient was age- and sex-matched with a healthy, non-dysphagic, community-dwelling adult retrieved from an extensive normative database.

\section{Methods}

\subsection{VFSS Selection}

This study was approved by our Institutional Review Board (Pro00067126) and conducted to conform within the Code of Ethics of the World Medical Association (Declaration of Helsinki). Eligible subjects were identified via electronic medical record review using current procedure terminology codes 22561 and 92611 to cross reference patients who underwent both ACSS and VFSS at our institution between January 1, 2010 and October 5, 
2018. Medical records were then reviewed to ensure patients had new onset of subjective complaints of dysphagia post-ACSS (e.g., food sticking, coughing during meals) prompting a referral for VFSS. Studies were completed in both the inpatient and outpatient settings using identical protocols. Only the initial VFSS ordered after onset of dysphagia symptoms for each patient was evaluated so that no repeat studies were included in data analysis. Patients with preexisting documented dysphagic complaints, altered diets or gastric tube placement were excluded. Once all VFSS records meeting this study inclusion criteria were obtained, these patients were categorized into two groups based on the duration of post-operative dysphagia. Patients in the "early" group presented for VFSS $\leq 2$ months post-surgery while patients in the "late" group had dysphagia that persisted $>2$ months post-surgery.

\subsection{Equipment and procedures}

All VFSS recordings included in the study followed the Modified Barium Swallow Impairment Profile (MBSImPTM) protocol, which includes 12 swallow tasks, with 10 swallow tasks captured in a lateral view and two in the anterior-posterior (AP) view. Regions of visualization include the oral cavity, pharynx, larynx, and esophagus. Patients are administered standardized, commercial preparations of barium contrast agents (VARIBAR $®$ barium sulfate $40 \%$ weight/volume; Bracco Diagnostics, Inc., Monroe Township, NJ) that include thin $(<15 \mathrm{cps})$ barium (two trials of $5 \mathrm{ml}$ via teaspoon, one cup sip [20 mL], and sequential swallows from cup [ $40 \mathrm{~mL}]$ ), nectar (150-450 cps) barium (one trial of $5 \mathrm{~mL}$ via teaspoon, one cup sip [20 mL], and sequential swallows from cup [ $40 \mathrm{~mL}])$, thin honey $(800-1800 \mathrm{cps})$ barium (one trial of $5 \mathrm{ml}$ via teaspoon), pudding (4500-7000 cps) barium (one trial of $5 \mathrm{~mL}$ via teaspoon), and one-half portion of a shortbread cookie (Lorna Doone, Nabisco, East Hanover, New Jersey, USA) coated with $3 \mathrm{~mL}$ pudding barium in the lateral view (Martin-Harris et al., 2008). The $5 \mathrm{~mL}$ nectar and pudding tasks are repeated in the AP viewing plane. Studies were obtained using continuous fluoroscopy and digital recordings were made with a resolution of 60 fields (30 frames) per second. Two separate videofluoroscopic recording devices (Digital Swallowing Workstation Model 7100, Kay Elemetrics Corp, Lincoln Park, New Jersey, USA; TIMS DICOM SP 2000 System, TIMS Medical, Chelmsford, Massachusetts, USA) were used for signal acquisition, digital storage, and retrieval of the swallowing data.

\subsection{Data collection and analysis}

MBSImP (Martin-Harris et al., 2008; MartinHarris et al., 2017) and Penetration Aspiration Scale (PAS) (Rosenbek et al., 1996) scores were abstracted from speech-language pathology (SLP) VFSS reports within the medical record. All SLPs complete standardized MBSImP training and testing with $a \geq 80 \%$ reliability pass rate and ongoing, quarterly calibration training to ensure that $\geq 80 \%$ reliability is maintained. The MBSImP tool includes 17 components of swallowing physiology across three functional domains: oral (Components 1-6), pharyngeal (Components 7-16), and esophageal (Component 17). MBSImP components are scored on an ordinal scale from 0 (indicating no impairment) to a maximum of 2,3 , or 4 , depending on the specific component (Martin-Harris et al., 2008; Martin-Harris et al., 2017). Higher scores indicate increasingly worse impairment. MBSImP Overall Impression (OI) scores, representing the worst (highest) score across all swallow tasks as appropriate, were extracted for the 10 physiologic components included within the pharyngeal domain: soft palate elevation, laryngeal elevation, anterior hyoid excursion, epiglottic movement, laryngeal vestibular closure, pharyngeal stripping wave, pharyngeal contraction, pharyngoesophageal segment opening, tongue base retraction and pharyngeal residue. OI scores were summed across all pharyngeal components according to MBSImP procedural guidelines to derive a Pharyngeal Total (PT) score (Martin-Harris et al., 2017).

The presence, depth and patient response to airway invasion were evaluated using the PenetrationAspiration Scale (PAS), which is a validated, eight-point ordinal scale (Rosenbek et al., 1996). A score of 1 represents the absence of airway invasion, and a score of 8 represents an absent patient reaction (cough) to aspirated material (i.e. silent aspiration). Based on previous literature examining PAS scores in healthy adults, scores of 3 and greater were considered impaired with 3-5 defined as penetration and scores 6-8 as aspiration (Robbins et al., 1999). We extracted the highest (worst) PAS score (maxPAS) across all swallow tasks in the lateral viewing plane for data analysis.

Posterior pharyngeal wall thickness (PWT) was measured on a single rest frame from the VFSS recording using ImageJ (National Institutes of 
Health, Bethesda, Maryland) using a penny placed on the patient's neck or known screw length from operative note as a reference scalar. PWT measurements were made at the thickest point of the posterior pharyngeal wall at the height of the base of the vallecula/epiglottis and at an angle perpendicular to the cervical spine (i.e. the vertical axis formed by the anterior edges of the cervical vertebral bodies) (Fig. 1). This site was selected for measurement because increased thickness of the pharynx at this level is most likely to interfere with epiglottic inversion and subsequent vallecular clearing (Leonard \& Belafsky, 2011). Interrater reliability testing was completed in a blinded fashion between two authors ( $\mathrm{RC}$ and $\mathrm{AO}$ ) and analyzed with using Pearson's correlation coefficient.

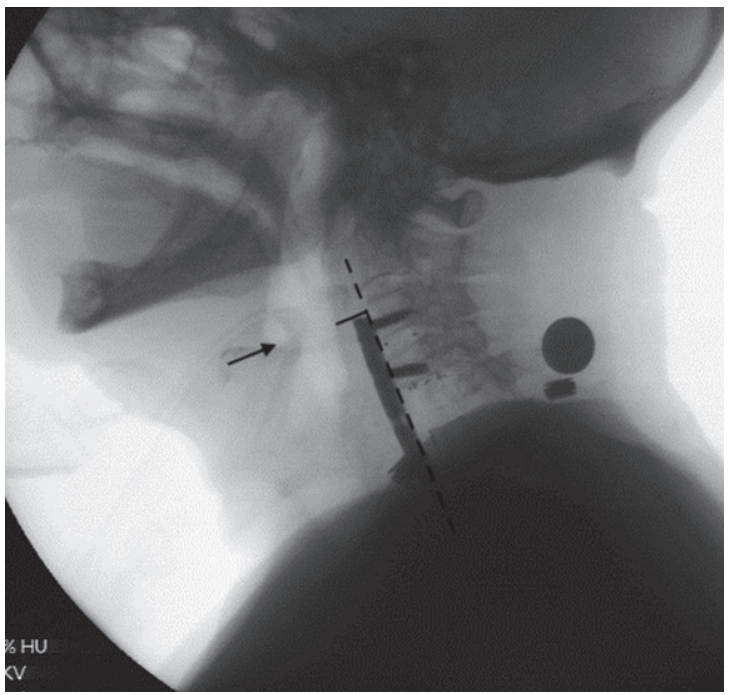

Fig. 1. Posterior pharyngeal wall thickness (PWT) was measured on a single rest frame from the VFSS recording using ImageJ (National Institutes of Health, Bethesda, Maryland) utilizing a penny placed on the patient's neck as a referent scalar. The solid line is the measurement taken perpendicular to the cervical spine axis (dotted line) at the level of the base of the vallecula/epiglottis (arrow).
Each eligible patient was then age- and sexmatched with a healthy, non-dysphagic, communitydwelling adult derived from a large normative database housing of 195 adults, ranging in age from 21 to 89 years.

All data analyses were performed by SigmaPlot 12.5 (Systat Software, Inc., San Jose, CA, USA) and SPSS 25.0 (IBM Corporation). All continuous variables were tested for normal distribution as determined by the Kolmogorov-Smirnov test. Categorical variables were summarized by frequency, percentage, or range. Continuous variables were summarized by mean (SD) or median (interquartile range) values where appropriate. For primary continuous outcome measures (MBSImP OI and PT scores, PAS scores, and PWT), comparisons among groups (early patient group, late patient group, and controls) to determine if differences existed between groups were conducted with a One-Way analysis of variance (ANOVA) or Kruskal-Wallis test where appropriate and followed by post-hoc Tukey comparison tests. Correlation and regression models were used to determine the relationship between the independent variables (demographics) and dependent outcome variables (scores) but these were not statistically significant due to lack of sample size. Power analyses at the probability level of 0.05 were done for a correlation model where a sample size of 75 would yield $44.5 \%$ power, and multiple regression (medium effect size of 0.15 ) with four independent predictors where a sample size of 75 would yield $21.2 \%$ power. A $p$-value of $<0.05$ was considered to indicate a statistically significant difference for all statistical tests.

\section{Results}

Seventy-five patients with new onset of dysphagic complaints following ACSS were included in the study (Table 1). The mean age of the early postoperative group was 61.5 years (range $=21-82$ years),

Table 1

Patient demographic and clinical information

\begin{tabular}{|c|c|c|c|}
\hline & \multicolumn{2}{|c|}{ ACSS Patients } & \multirow[b]{2}{*}{$\begin{array}{c}\text { Controls } \\
(n=75)\end{array}$} \\
\hline & $\begin{array}{c}\text { Early } \\
(n=57)\end{array}$ & $\begin{array}{c}\text { Late } \\
(n=18)\end{array}$ & \\
\hline Mean age, years (Range; SD) & $61.5(21-82 ; 12.4)$ & $52.6(29-69 ; 11.3)$ & $58.1(24-86 ; 13.2)$ \\
\hline Mean time from surgery, days (Range; SD) & $11.1(1-60 ; 12.4)$ & $340.5(76-1119 ; 305.2)$ & N/A \\
\hline Mean vertebral levels involved (Range; SD) & $2.1(1-4 ; 1)$ & $1.9(1-3 ; 0.9)$ & N/A \\
\hline \multicolumn{4}{|l|}{$\operatorname{Sex}, n(\%)$} \\
\hline Male & $34(59.7)$ & $7(38.9)$ & $41(54.7)$ \\
\hline Female & $23(40.4)$ & $11(61.1)$ & $34(45.3)$ \\
\hline
\end{tabular}


with $40 \%(n=23)$ comprised of females. The late post-operative group had a mean age of 52.6 years (range $=29-69$ years), with majority female $(61 \%$, $n=11)$. Therefore, the mean age of the early postop group was significantly higher than the late group $(P=0.01)$, although gender distribution was comparable between groups $(P=0.12)$. Most patients $(76 \%$, $n=57)$ were within 2 months following surgery $($ mean $=11.1$ days, range $=1-60$ days $)$, while 18 patients $(24 \%)$ were greater than 2 months postsurgery (mean $=340.5$ days, range $=76$ days -1119 days). The mean number of vertebral levels involved in the early group was 2.1 (range $=1-4, \mathrm{C} 2-\mathrm{T} 1)$ and was 1.9 in the late group (range $=1-3, \mathrm{C} 3-\mathrm{T} 1$ ). The number of levels addressed during surgery did not vary significantly between groups $(P=0.43)$. Overall, significant differences were identified for all evaluated parameters of interest (e.g., MBSImP PT scores, PAS scores, and PWT) between control subjects and the early post-op group (Table 2).

Table 2

Primary outcomes of interest between controls, early post-operative and late post-operative patients

\begin{tabular}{lcccc}
\hline Measure & Group & Mdn & IQR & $P$-value \\
\hline PAS Score & $0^{\mathrm{a}}$ & 1.00 & $1.00 / 2.00$ & 0 to $1, P=5.1 \mathrm{e}-14^{*}$ \\
& $1^{\mathrm{b}}$ & 7.00 & $3.00 / 8.00$ & 0 to $2, P=1.00$ \\
PT Score & $2^{\mathrm{c}}$ & 1.00 & $1.00 / 1.25$ & 1 to $2, P=3.6 \mathrm{e}-6^{*}$ \\
& 0 & 5.00 & $4.00 / 7.00$ & 0 to $1, P=2.3 \mathrm{e}-14^{*}$ \\
& 1 & 12.00 & $9.00 / 14.00$ & 0 to $2, P=0.70$ \\
PWT $(\mathrm{mm})$ & 2 & 2.00 & $0.75 / 7.25$ & 1 to $2, P=5.5 \mathrm{e}-6^{*}$ \\
& 0 & 4.68 & $3.81 / 5.41$ & 0 to $1, P=0^{*}$ \\
& 1 & 14.32 & $11.12 / 17.33$ & 0 to $2, P=2.5 \mathrm{e}-5^{*}$ \\
& 2 & 9.15 & $5.31 / 13.01$ & 1 to $2, P=0.07$ \\
\hline
\end{tabular}

PAS, penetration-aspiration scale; PT, pharyngeal total; PWT, pharyngeal wall thickness. ${ }^{\mathrm{a}}$ Group $0=$ controls. ${ }^{\mathrm{b}}$ Group $1=$ early post-operative patients $(\leq 2 \mathrm{mo})$. ${ }^{\mathrm{c}}$ Group $2=$ late post-operative patients $(>2 \mathrm{mo}) .{ }^{*}=$ Denotes significance at $P<0.05$.

\subsection{MBSImP PT scores}

MBSImP PT scores were significantly greater in the early postop group (IQR $=9-14$, median $=12$ ) compared to the control group $(4-7,5, P<0.001)$ and late postop group $(0.75-7.25,2, P<0.001)$. The difference in PT scores between the late postop group and controls was not statistically significant $(P=0.70)$. The frequency of MBSImP OI scores distributed across the pharyngeal domain were recorded (Table 3).

\subsection{PAS scores}

The median maxPAS score in the early postoperative group $(n=57)$ was 7 , with aspiration $(\mathrm{PAS}>6)$ observed in the majority of patients $(60 \%$; $n=34)$. The median maxPAS score in the late post-operative group ( $n=18)$ was 1 , with aspiration (PAS $>6)$ rarely observed $(5.6 \% ; n=1)$. Penetration (PAS $=3-5)$ was also seen frequently in the early post-operative group $(19.3 \% ; n=11)$ but was not observed in the late-post operative group. Thus, the early postoperative group ( $\mathrm{IQR}=3-8$, median $=7$ ) had significantly higher maxPAS scores than both the control group $(1-2,1, P<0.001)$ and late postoperative group $(1-1.25,1, P<0.001)$. There was no significant difference in maxPAS scores between the late post-operative group and controls $(P=1.00)$.

\section{3. $P W T$}

Median PWT was $4.68 \mathrm{~mm}$ in the control group $(\mathrm{IQR}=3.81-5.41 \mathrm{~mm})$, compared to $14.32 \mathrm{~mm}$ in the early post-operative group $(11.12-17.33, P<0.001)$ and $9.15 \mathrm{~mm}$ in the late post-operative group (5.31-13.01, $P<0.001)$. PWT interrater reliability

Table 3

Frequency of Modified Barium Swallow Impairment Profile (MBSImP) Overall Impression scores distributed across the pharyngeal domain.

\begin{tabular}{|c|c|c|c|c|c|}
\hline \multirow[t]{2}{*}{ MBSImP Component } & \multicolumn{5}{|c|}{$\begin{array}{l}\text { Overall impression (OI) score Frequency } \\
\text { (Percentage) }\end{array}$} \\
\hline & 0 & 1 & 2 & 3 & 4 \\
\hline 7-Soft palate elevation & $62(82.7)$ & $12(16)$ & $0(0)$ & $0(0)$ & $1(1.3)$ \\
\hline 8-Laryngeal elevation & $23(30.7)$ & $33(44)$ & $19(25.3)$ & $0(0)$ & \\
\hline 9-Anterior hyoid excursion & $20(26.7)$ & $47(62.7)$ & $8(10.7)$ & & \\
\hline 10-Epiglottic movement & $20(26.7)$ & $19(25.3)$ & $36(48)$ & & \\
\hline 11-Laryngeal vestibular closure-height of swallow & $24(32)$ & $41(54.7)$ & $10(13.3)$ & & \\
\hline 12-Pharyngeal stripping wave & $20(26.7)$ & $38(50.7)$ & $17(22.7)$ & & \\
\hline 13-Pharyngeal contraction* (AP view only) & $34(45.3)$ & $4(5.3)$ & $4(5.3)$ & $6(8)$ & \\
\hline 14-Pharyngoesophageal segment opening & $12(16)$ & $38(50.7)$ & $22(29.3)$ & $3(4)$ & \\
\hline 15-Tongue base retraction & $11(14.7)$ & $24(32)$ & $32(42.7)$ & $8(10.7)$ & $0(0)$ \\
\hline 16-Pharyngeal residue & $2(2.7)$ & $14(18.7)$ & $38(50.7)$ & $19(25.3)$ & $2(2.7)$ \\
\hline
\end{tabular}

* Missing $N=27$. 
testing revealed strong agreement with $r=0.81(95 \%$ confidence interval 0.393 to $0.956 ; P=0.004$ ). There was no significant decrease in PWT measurements between the late post-operative group and the early post-operative group $(P=0.07)$. Regression analysis revealed the correlation between days post-surgery and PWT to be $r=-0.229(P=0.056)$.

\section{Discussion}

The purpose of this investigation was to examine post-operative changes in structural, physiologic, and airway invasion measures in patients with subjective dysphagia symptoms at different timepoints following ACSS compared to healthy controls. We found that patients in the early phase ( $\leq 2$ months) of recovery exhibited significant structural and physiologic pharyngeal swallowing impairment as well as a higher incidence of bolus airway invasion on VFSS as compared to healthy controls and patients in the late phase of recovery (> 2 months). Our findings agree with previous investigations that have revealed a decrease in dysphagic symptoms within the first two months post-ACSS with most patients able to return to normal diets shortly after (Leonard \& Belafsky, 2011; Kang et al., 2011; Miles et al., 2019; Yu \& Tao, 2020).

Several studies have attributed initial post-ACSS swallowing dysfunction to a variety of etiologies with soft tissue edema being the most common (Leonard \& Belafsky, 2011; Kang et al., 2011; Yang et al., 2012; Min et al., 2016). It has been hypothesized that during the acute recovery phase, the progressive decrease in swelling allows for more complete clearing of the vallecular space and pyriform sinuses, ultimately resulting in resolution of symptoms. However, we found that pharyngeal wall thickness in the late post-operative ACSS group was nearly twice that of controls and this finding is consistent with the literature as well (Muss et al., 2017; Leonard \& Belafsky, 2011; Miles et al., 2019; Daggett et al., 2006; Khaki et al., 2013). Since the late group displayed a wide range of days post-surgery (76-1119 days), correlational analysis was completed. There was a weak, although non-significant, negative correlation between days post-surgery and PWT $(r=-0.229$; $P=0.056)$. The question remains at what timepoint PWT returns to baseline, if at all, which is beyond the scope of this manuscript as we did not follow patients longitudinally over time. Nonetheless, it is interesting, that our results revealed that chronic increased PWT was not associated with concurrent physiologic pharyngeal impairment to explain subjective dysphagia symptoms.

Although subjective complaints of dysphagia had resulted in referral for VFSS, the findings on VFSS for late post-ACSS patients were not significantly different compared to healthy controls. Kang et al. (2016) also found similar disagreement in a recent prospective study exploring post-surgical dysphagia following ACSS. At four weeks post-surgery, 3 of 9 patients with subjective dysphagia symptoms had normal VFSS results defined by absence of pharyngeal residue, overt penetration, and aspiration. In contrast, they also found that $50 \%$ of patients who did not have dysphagia symptoms at four weeks postACSS had abnormal VFSS results (Kang et al., 2016). This begs the question of the source of subjective dysphagia symptoms and the accuracy of our standard assessments in late post-ACSS patients. The differences could be related to subtle contractility deficits, sensory impairments, or mechanical alterations in spinal movement during swallowing. Pre and post-operative kinematic studies and high resolution pharyngeal manometry may be more revealing to assess these changes that patients perceive but our gold standard assessments fail to measure.

ACSS may also create neurogenic dysphagia by injury to the recurrent laryngeal nerve (CN X), superior laryngeal nerve $(\mathrm{CN} \mathrm{X})$, glossopharyngeal nerve (CN IX) or the pharyngeal plexus (CN X, CN IX) but the majority of these are thought to be traction injuries that should improve over time. With persistent neurologic injury, we would expect to see impairment in delayed initiation of pharyngeal swallow (MBSImP Component 6), pharyngeal stripping wave (Component 12), and pharyngeal contraction (Component 13). However, due to limitation in sample size, we were unable to convert total impairment PT scores from a continuous variable to a categorical variable to perform logistic regression. This information may have helped inform us on which components of pharyngeal dysfunction contributed most to pharyngeal dysfunction (i.e., impaired pharyngeal contraction, delayed initiation of pharyngeal swallow, etc.).

Possible anatomic causes of post-ACSS dysphagia are often overlooked, including acute surgically induced changes in spine orientation (e.g. straightening of the natural lordosis of the cervical spine), loss of range of motion and/or presence of a potentially obstructive foreign body. These changes may alter the perception of swallowing physiology in a way that traditional VFSS parameters cannot measure (Tian \& Yu, 2013; Tian \& Yu, 2017; Radcliff et al., 2016), 
leading to mismatch in subjective complaints versus objective findings. Unpublished data from our institution has shown a high incidence of decreased epiglottic inversion (Component 10) due to spinal hardware or osteophytic obstruction at $\mathrm{C} 2-\mathrm{C} 4$ level. This is supported by another study of 24 patients using zero profile devices for multi-level discectomy and fusion who exhibited the expected incidence of early postoperative dysphagia but no subjective dysphagic complaints at $>6$ months (Albanese et al., 2017).

The limitations of our study include the retrospective nature, a relatively small sample size, and an unequal distribution in the number of subjects among the early and late postoperative groups. There was also a significant difference in age between the early and late groups (mean $=61.5$ vs. 52.6 , respectively, $P=0.01$ ), potentially limiting the strength of our analyses comparing the two. Further, reliability for MBSImP and PAS scores was not ascertained. However, all speech-language pathologists are required to maintain MBSImP certification, which means that they have demonstrated initial $\geq 80 \%$ reliability and undergo quarterly calibration training to ensure $\geq 80 \%$ reliability is maintained for scoring the parameters evaluated in this study. Another potential limitation of our study was the large range of days following ACSS surgery seen in the late group. The decision to a priori dichotomize the groups at two months was based on previous studies that have utilized similar metrics (Leonard \& Belafsky, 2011; Miles et al., 2019). The study was also limited by our sampling method, which selected only for ACSS patients who were referred for VFSS due to subjective complaints of dysphagia. This is a form of sampling bias and therefore limits generalization across all ACSS patients. Finally, a swallow task may (e.g., solid/cookie trial) have been omitted to ensure patient safety. In these situations, clinicians abided by the standardized MBSImP bailout scoring rules in order to maintain a high level of internal validity.

\section{Conclusion}

Subjective complaints of dysphagia can persist for more than two months following ACSS, but often do not correlate with validated physiologic swallowing dysfunction on VFSS. This can be a frustrating situation for patients due to a lack of therapeutic targets for intervention and no specific explanation for their complaints. Future studies should focus on potential applications of newer technology to elucidate relevant deficits.

\section{Acknowledgments}

The authors have no acknowledgments.

\section{Conflict of interest}

The authors have no conflicts of interest to report. Given her role as an Editorial Board Member, Ashli K. O'Rourke had no involvement nor access to information regarding the peer review of this article.

\section{References}

Rihn, J.A., Kane, J., Albert, T.J., Vaccaro, A.R., \& Hilibrand, A.S. (2011). What is the incidence and severity of dysphagia after anterior cervical surgery? Clinical Orthopaedics and Related Research, 469(3), 658-665. https://doi.org/10.1007/s11999010-1731-8

Anderson, K.K., \& Arnold, P.M. (2013). Oropharyngeal Dysphagia after anterior cervical spine surgery: a review. Global Spine Journal, 3(4), 273-286. https://doi.org/10.1055/s-0033-13 54253

Liu, F.Y., Yang, D.L., Huang, W.Z., Huo, L.S., Ma, L., Wang, H., Yang, S.D., \& Ding, W.Y. (2017). Risk factors for dysphagia after anterior cervical spine surgery: A meta-analysis. Medicine, 96(10), e6267. https://doi.org/10.1097/MD.000000 0000006267

Frempong-Boadu, A., Houten, J.K., Osborn, B., Opulencia, J., Kells, L., Guida, D.D., \& Le Roux, P.D. (2002). Swallowing and speech dysfunction in patients undergoing anterior cervical discectomy and fusion: a prospective, objective preoperative and postoperative assessment. Journal of Spinal Disorders \& Techniques, 15(5), 362-368. https://doi.org/10.1097/00024 720-200210000-00004

Kang, S.H., Kim, D.K., Seo, K.M., Lee, S.Y., Park, S.W., \& Kim, Y.B. (2016). Swallowing Function Defined by Videofluoroscopic Swallowing Studies after Anterior Cervical Discectomy and Fusion: a Prospective Study. Journal of Korean Medical Science, 31(12), 2020-2025. https://doi.org/10.3346/jkms. 2016.31.12.2020

Kilburg, C., Sullivan, H.G., \& Mathiason, M.A. (2006). Effect of approach side during anterior cervical discectomy and fusion on the incidence of recurrent laryngeal nerve injury. Journal of Neurosurgery. Spine, 4(4), 273-277. https://doi.org/10.3171/ spi.2006.4.4.273

Beutler, W.J., Sweeney, C.A., \& Connolly, P.J. (2001). Recurrent laryngeal nerve injury with anterior cervical spine surgery risk with laterality of surgical approach. Spine, 26(12), 1337-1342. https://doi.org/10.1097/00007632-200106150-00014

Badhiwala, J.H., Nassiri, F., Witiw, C.D., Mansouri, A., Almenawer, S.A., da Costa, L., Fehlings, M.G., \& Wilson, J.R. (2019). Investigating the utility of intraoperative neurophysiological monitoring for anterior cervical discectomy and fusion: analysis of over 140,000 cases from the National (Nationwide) Inpatient Sample data set. Journal of Neurosurgery. Spine, 31(1), 76-86. https://doi.org/10.3171/2019.1.SPINE181110

Shriver, M.F., Lewis, D.J., Kshettry, V.R., Rosenbaum, B.P., Benzel, E.C., \& Mroz, T.E. (2017). Dysphagia Rates after Anterior 
Cervical Diskectomy and Fusion: A Systematic Review and Meta-Analysis. Global Spine Journal, 7(1), 95-103. https:// doi.org/10.1055/s-0036-1583944

Riley, L.H., 3rd, Vaccaro, A.R., Dettori, J.R., \& Hashimoto, R. (2010). Postoperative dysphagia in anterior cervical spine surgery. Spine, 35(9 Suppl), S76-S85. https://doi.org/10.1097/ BRS.0b013e3181d81a96

Bazaz, R., Lee, M.J., \& Yoo, J.U. (2002). Incidence of dysphagia after anterior cervical spine surgery: a prospective study. Spine, 27(22), 2453-2458. https://doi.org/10.1097/0000 7632-200211150-00007

Belafsky, P.C., Mouadeb, D.A., Rees, C.J., Pryor, J.C., Postma, G.N., Allen, J., \& Leonard, R.J. (2008). Validity and reliability of the Eating Assessment Tool (EAT-10). The Annals of Otology, Rhinology, and Laryngology, 117(12), 919-924. https:// doi.org/10.1177/000348940811701210

Rosenthal, B.D., Nair, R., Hsu, W.K., Patel, A.A., \& Savage, J.W. (2016). Dysphagia and Dysphonia Assessment Tools After Anterior Cervical Spine Surgery. Clinical Spine Surgery, 29(9), 363-367. https://doi.org/10.1097/BSD.0000000000000373

Wilmskoetter, J., Bonilha, H., Hong, I., Hazelwood, R.J., MartinHarris, B., \& Velozo, C. (2019). Construct validity of the Eating Assessment Tool (EAT-10). Disability and Rehabilitation, 41(5), 549-559. https://doi.org/10.1080/09638288.2017. 1398787

Muss, L., Wilmskoetter, J., Richter, K., Fix, C., Stanschus, S., Pitzen, T., Drumm, J., \& Molfenter, S. (2017). Changes in Swallowing After Anterior Cervical Discectomy and Fusion With Instrumentation: A Presurgical Versus Postsurgical Videofluoroscopic Comparison. Journal of Speech, Language, and Hearing Research : JSLHR, 60(4), 785-793. https://doi.org/ 10.1044/2016_JSLHR-S-16-0091

Martin-Harris, B., Brodsky, M.B., Michel, Y., Castell, D.O., Schleicher, M., Sandidge, J., Maxwell, R., \& Blair, J. (2008). MBS measurement tool for swallow impairment-MBSImp: establishing a standard. Dysphagia, 23(4), 392-405. https://doi.org/ 10.1007/s00455-008-9185-9

Martin-Harris, B., Logemann, J.A., McMahon, S., Schleicher, M., \& Sandidge, J. (2000). Clinical utility of the modified barium swallow. Dysphagia, 15(3), 136-141. https://doi.org/10.1007/ s004550010015

Martin, R.E., Neary, M.A., \& Diamant, N.E. (1997). Dysphagia following anterior cervical spine surgery. Dysphagia, 12(1), 2-10. https://doi.org/10.1007/p100009513

Leonard, R., \& Belafsky, P. (2011). Dysphagia following cervical spine surgery with anterior instrumentation: evidence from fluoroscopic swallow studies. Spine, 36(25), 2217-2223. https:// doi.org/10.1097/BRS.0b013e318205a1a7

Smith-Hammond, C.A., New, K.C., Pietrobon, R., Curtis, D.J., Scharver, C.H., \& Turner, D.A. (2004). Prospective analysis of incidence and risk factors of dysphagia in spine surgery patients: comparison of anterior cervical, posterior cervical, and lumbar procedures. Spine, 29(13), 1441-1446. https:// doi.org/10.1097/01.brs.0000129100.59913.ea

Martin-Harris, B., Humphries, K., \& Garand, K.L. (2017). The modified barium swallow impairment profile (MBSImPTMC)innovation, dissemination and implementation. Perspectives of the ASHA Special Interest Groups, 2(13), 129-138.

Rosenbek, J.C., Robbins, J.A., Roecker, E.B., Coyle, J.L., \& Wood, J.L. (1996). A penetration-aspiration scale. Dysphagia, 11(2), 93-98. https://doi.org/10.1007/BF00417897
Robbins, J., Coyle, J., Rosenbek, J., Roecker, E., \& Wood, J. (1999). Differentiation of normal and abnormal airway protection during swallowing using the penetration-aspiration scale. Dysphagia, 14(4), 228-232. https://doi.org/10.1007/ PL00009610

Kang, S.H., Kim, D.K., Seo, K.M., Kim, K.T., \& Kim, Y.B. (2011). Multi-level spinal fusion and postoperative prevertebral thickness increase the risk of dysphagia after anterior cervical spine surgery. Journal of Clinical Neuroscience : Official Journal of the Neurosurgical Society of Australasia, 18(10), 1369-1373. https://doi.org/10.1016/j.jocn.2011.02.033

Miles, A., Jamieson, G., Shasha, L., \& Davis, K. (2019). Characterizing dysphagia after spinal surgery. The Journal of Spinal Cord Medicine, 1-9. Advance online publication. https:// doi.org/10.1080/10790268.2019.1665613

Yu J, \& Tao X. (2020). Incidence and Risk Factors of postoperative Dysphagia following Cervical Spine Surgery. PREPRINT (Version 1) available at Research Square. https:// doi:10.21203/rs.3.rs-46131/v1

Yang, L., Gu, Y., Liang, L., Gao, R., Shi, S., Shi, J., \& Yuan, W. (2012). Stand-alone anchored spacer versus anterior plate for multilevel anterior cervical diskectomy and fusion. Orthopedics, 35(10), e1503-e1510. https://doi.org/10.3928/0147 7447-20120919-20

Min, Y., Kim, W.S., Kang, S.S., Choi, J.M., Yeom, J.S., \& Paik, N.J. (2016). Incidence of Dysphagia and Serial Videofluoroscopic Swallow Study Findings After Anterior Cervical Discectomy and Fusion: A Prospective Study. Clinical Spine Surgery, 29(4), E177-E181. https://doi.org/10.1097/BSD.000000000 0000060

Daggett, A., Logemann, J., Rademaker, A., \& Pauloski, B. (2006). Laryngeal penetration during deglutition in normal subjects of various ages. Dysphagia, 21(4), 270-274. https://doi.org/ 10.1007/s00455-006-9051-6

Khaki, F., Zusman, N.L., Nemecek, A.N., Ching, A.C., Hart, R.A., \& Yoo, J.U. (2013). Postoperative prevertebral soft tissue swelling does not affect the development of chronic dysphagia following anterior cervical spine surgery. Spine, 38(9), E528E532. https://doi.org/10.1097/BRS.0b013e31828a2992

Tian, W., \& Yu, J. (2013). The role of C2-C7 and O-C2 angle in the development of dysphagia after cervical spine surgery. Dysphagia, 28(2), 131-138. https://doi.org/10.1007/s00455012-9421-1

Tian, W., \& Yu, J. (2017). The Role of C2-C7 Angle in the Development of Dysphagia After Anterior and Posterior Cervical Spine Surgery. Clinical Spine Surgery, 30(9), E1306-E1314. https://doi.org/10.1097/BSD.0000000000000493

Radcliff, K.E., Bennett, J., Stewart, R.J., Kepler, C.K., Sidhu, G.S., Hilibrand, A.S., Kane, J., Albert, T.J., Vaccaro, A.R., \& Rihn, J.A. (2016). Change in Angular Alignment Is Associated With Early Dysphagia After Anterior Cervical Discectomy and Fusion. Clinical Spine Surgery, 29(6), 248-254. https:// doi.org/10.1097/BSD.0b013e31828b39b4

Albanese, V., Certo, F., Visocchi, M., \& Barbagallo, G. (2017). Multilevel Anterior Cervical Diskectomy and Fusion with Zero-Profile Devices: Analysis of Safety and Feasibility, with Focus on Sagittal Alignment and Impact on Clinical Outcome: Single-Institution Experience and Review of Literature. World Neurosurgery, 106, 724-735. https://doi.org/10.1016/ j.wneu.2017.06.051 